\title{
A NOTE ON QUADRICS THROUGH AN ALGEBRAIC CURVE
}

\author{
FERNANDO SERRANO
}

\author{
(Communicated by William C. Waterhouse)
}

\begin{abstract}
In this note we describe the intersection of all quadric hypersurfaces containing a given linearly normal smooth projective curve of genus $n$ and degree $2 n+1$.
\end{abstract}

Let $C$ be an irreducible nonsingular curve of genus $g$, defined over an algebraically closed field of any characteristic. Let $C$ be embedded in $\mathbf{P}^{r}$ by a complete linear system $|L|$. Saint-Donat [5] has proved that if $\operatorname{deg} L \geq 2 g+2$ then the homogeneous ideal $I_{C}$ of $C \subseteq \mathrm{P}^{r}$ is generated by quadrics, and if $\operatorname{deg} L=2 g+1$ then $I_{C}$ is generated by quadrics and cubics (see also Fujita [1]). In [2], Green and Lazarsfeld have announced the following result: In case $\operatorname{deg} L=2 g+1, I_{C}$ fails to be generated by quadrics if and only if $C$ is hyperelliptic or $L$ embeds $C$ with a trisecant line, i.e., $H^{0} O_{C}\left(L-K_{C}\right) \neq 0$, where $K_{C}$ denotes the canonical divisor on $C$. In this note we describe the intersection of all quadric hypersurfaces passing through $C \subseteq \mathbb{P}^{r}$ in the borderline situation $\operatorname{deg} L=2 g+1$. The main ingredient of the proof is a theorem of Castelnuovo on the postulation of points.

A $g_{d}^{1}$ on a curve is, by definition, a base-point free linear system of degree $d$ and dimension 1. For the definition and properties of rational normal scrolls see [3].

Our result is the following.

THEOREM. Let $C \subseteq \mathbf{P}^{n+1}$ be a linearly normal smooth irreducible curve of genus $n \geq 4$ and degree $2 n+1$. If $W(C)$ denotes the intersection of all quadric hypersurfaces of $\mathbf{P}^{n+1}$ which contain $C$, then either $W(C)$ consists of $C$ plus (possibly) a line and finitely many isolated points, or $W(C)$ is a rational normal scroll of dimension 2. In case $W(C)$ is a scroll, one of the following situations occurs:

(i) $W(C)$ is smooth and $C$ meets every fiber of $W(C)$ at three points. $C$ is trigonal and embedded by the linear system $\left|K_{C}+g_{3}^{1}\right|$.

(ii) $W(C)$ is a cone with vertex $P$, and $C$ passes through $P$ and meets every fiber of $W(C)$ at $P$ plus two other points. $C$ is hyperelliptic and embedded by $\left|P+n g_{2}^{1}\right|$.

(iii) $W(C)$ is smooth and $C$ is a divisor in $W(C)$ of class $2 H+R$, where $H$ denotes a hyperplane and $R$ a fiber of the ruling. In particular $C$ is hyperelliptic, the $g_{2}^{1}$ being given by restriction of the ruling of $W(C)$.

PrOOF. Throughout this proof we will assume that $W(C)$ is not the union of $C$ and (possibly) a line plus finitely many points. Consequently, there exists a curve $G \subseteq W(C), G \neq C$, with degree of $G \geq 2$. $G$ is allowed to be a pair of distinct lines. Pick two distinct general points $Q_{1}$ and $Q_{2}$ on $G$, none of them on $C$. If $G$ is a

Received by the editors October 20, 1986.

1980 Mathematics Subject Classification (1985 Revision). Primary 14E25, 14H45, 14 N05.

Key words and phrases. Rational scroll, hyperelliptic, trigonal.

(C) 1988 American Mathematical Society $0002-9939 / 88 \$ 1.00+\$ .25$ per page 
union of two lines, then by a general pair we mean that $Q_{1}$ is a general point on one of the lines and $Q_{2}$ is general on the other line. Choose now a general hyperplane $\mathbf{P}^{n}$ in $\mathbf{P}^{n+1}$ passing through $Q_{1}$ and $Q_{2}$, and set $\Gamma=C \cap \mathbf{P}^{n}$. Let $W(\Gamma)$ be the intersection of all quadric hypersurfaces of $\mathbf{P}^{n}$ which contain $\Gamma$. If $I_{C}, I_{\Gamma}$ denote the ideal sheaves of $C$ in $\mathbf{P}^{n+1}$ and $\Gamma$ in $\mathbf{P}^{n}$ respectively, then the exact sequence

$$
\begin{aligned}
0= & H^{0}\left(\mathbf{P}^{n+1}, I_{C}(1)\right) \rightarrow H^{0}\left(\mathbf{P}^{n+1}, I_{C}(2)\right) \\
& \rightarrow H^{0}\left(\mathbf{P}^{n}, I_{\Gamma}(2)\right) \rightarrow H^{1}\left(\mathbf{P}^{n+1}, I_{C}(1)\right)=0
\end{aligned}
$$

yields $W(\Gamma)=W(C) \cap \mathbf{P}^{n}$.

ClAIM 1. $\Gamma$ consists of $2 n+1$ points in general linear position (i.e., any subset of $n+1$ points of $\Gamma$ spans $\left.\mathbf{P}^{n}\right)$.

PROOF OF Claim 1. Let $\left(\mathbf{P}^{n+1}\right)^{*}$ be the space of hyperplanes of $\mathbf{P}^{n+1}$. It is a well-known fact that the set

$$
\mathcal{U}=\left\{H \in\left(\mathbf{P}^{n+1}\right)^{*} \mid H \cap C \text { is in general linear position }\right\}
$$

is dense in $\left(\mathbf{P}^{n+1}\right)^{*}$. For $i=1,2$, the set $M\left(Q_{i}\right)=\left\{H \in\left(\mathbf{P}^{n+1}\right)^{*} \mid Q_{i} \in H\right\}$ is a hyperplane of $\left(\mathbf{P}^{n+1}\right)^{*}$. Since degree of $G \geq 2$ we have

$$
\bigcup_{Q_{1}, Q_{2} \in G}\left(M\left(Q_{1}\right) \cap M\left(Q_{2}\right)\right)=\left(\mathbf{P}^{n+1}\right)^{*}
$$

and thus $M\left(Q_{1}\right) \cap M\left(Q_{2}\right) \cap \mathcal{U} \neq \varnothing$ for a generic choice of $Q_{1}$ and $Q_{2}$. This proves Claim 1.

Choose linear subvarieties $\hat{\mathbf{P}}^{n-1}, \hat{\mathbf{P}}^{n}$ of $\mathbf{P}^{n+1}$ of dimensions $n-1$ and $n$ respectively. Let $\pi: C \rightarrow \hat{\mathbf{P}}^{n-1}$ be the projection of $C$ from the line $\overline{Q_{1} Q_{2}}$ spanned by $Q_{1}$ and $Q_{2}$, and let $\pi_{1}: C \rightarrow \hat{\mathbf{P}}^{n}$ be the projection of $C$ from $Q_{1}$.

CLAIM 2. $\pi$ and $\pi_{1}$ are generically one-to-one.

PROOF OF ClaIM 2. It suffices to prove the statement for $\pi$. Since $n+1 \geq 5$, any hyperplane passing through $Q_{1}$ and $Q_{2}$ contains at least three fibers of $\pi$. If $\pi$ has degree $k \geq 2$ then those three fibers consist of $3 k \geq 6$ points which span a $\mathbf{P}^{3}$ or a $\mathbf{P}^{4}$, so that they are not in general linear position. But this contradicts Claim 1 .

ClaIM 3. A general hyperplane of $\hat{\mathbf{P}}^{n}$ passing through the point $\overline{Q_{1} Q_{2}} \cap \hat{\mathbf{P}}^{n}$ cuts $\pi_{1}(C)$ at a set of points in general linear position.

ProOF OF Claim 3. We argue as in Claim 1. The set

$$
\mathcal{U}^{\prime}=\left\{H \in\left(\hat{\mathbf{P}}^{n}\right)^{*} \mid H \cap \pi_{1}(C) \text { is in general linear position }\right\}
$$

is dense in $\left(\hat{\mathbf{P}}^{n}\right)^{*}$, and $N\left(Q_{2}\right)=\left\{H \in\left(\hat{\mathbf{P}}^{n}\right)^{*} \mid \overline{Q_{1} Q_{2}} \cap \hat{\mathbf{P}}^{n} \in H\right\}$ is a hyperplane of $\left(\hat{\mathbf{P}}^{n}\right)^{*}$. Fix $Q_{1}$. Since $\operatorname{deg} G \geq 2$, the points $\overline{Q_{1} Q_{2}} \cap \hat{\mathbf{P}}^{n}$ describe a curve in $\hat{\mathbf{P}}^{n}$ as $Q_{2}$ varies along $G$. Therefore

$$
\left(\hat{\mathbf{P}}^{n}\right)^{*}=\bigcup_{Q_{2} \in G} N\left(Q_{2}\right)
$$

and thus $N\left(Q_{2}\right) \cap U^{\prime}=\varnothing$ for at most finitely many $Q_{2}$ 's.

ClaIM 4. $\Gamma \cup\left\{Q_{1}, Q_{2}\right\}$ is in general linear position in $\mathbf{P}^{n}$.

PROOF OF ClAIM 4. Choose any subset $\Omega$ of $n+1$ points in $\Gamma \cup\left\{Q_{1}, Q_{2}\right\}$. We have to show that $\Omega$ spans $\mathbf{P}^{n}$.

Case $1 . \Omega \subseteq \Gamma$. The claim is obvious because $\Gamma$ is in general linear position. 
Case 2. $\Omega=\left\{Q_{1}, T_{1}, \ldots, T_{n}\right\}$ with $\left\{T_{1}, \ldots, T_{n}\right\} \subseteq \Gamma$. By Claim 3, a general hyperplane $\mathbf{P}^{n} \subseteq \mathbf{P}^{n+1}$ containing $\overline{Q_{1} Q_{2}}$ cuts $\hat{\mathbf{P}}^{n}$ along an $(n-1)$-plane $\tilde{\mathbf{P}}^{n-1}$ such that $\tilde{\mathbf{P}}^{n-1} \cap \pi_{1}(C)$ is in general linear position. By Claim $2, \pi_{1}\left(T_{1}\right), \ldots, \pi_{1}\left(T_{n}\right)$ are all distinct and belong to $\tilde{\mathbf{P}}^{n-1} \cap \pi_{1}(C)$. Since $\left\{\pi_{1}\left(T_{1}\right), \ldots, \pi_{1}\left(T_{n}\right)\right\}$ spans $\tilde{\mathbf{P}}^{n-1}$, it follows that $\left\{Q_{1}, \pi_{1}\left(T_{1}\right), \ldots, \pi_{1}\left(T_{n}\right)\right\}$ spans $\mathbf{P}^{n}$, and so does $\Omega$.

Case 3. $\Omega=\left\{Q_{1}, Q_{2}, T_{1}, \ldots, T_{n-1}\right\}$ with $\left\{T_{1}, \ldots, T_{n-1}\right\} \subseteq \Gamma$. If $\tilde{\mathbf{P}}^{n-2}=\hat{\mathbf{P}}^{n-1} \cap$ $\mathbf{P}^{n}$ then $\tilde{\mathbf{P}}^{n-2} \cap \pi(C)$ is in general linear position. The points $\pi\left(T_{1}\right), \ldots, \pi\left(T_{n-1}\right)$ are all distinct because of Claim 2, and they belong to $\tilde{\mathrm{P}}^{n-2} \cap \pi(C)$. Inasmuch as $\left\{\pi\left(T_{1}\right), \ldots, \pi\left(T_{n-1}\right)\right\}$ spans $\tilde{\mathbf{P}}^{n-2}$ we get that $\left\{Q_{1}, Q_{2}, \pi\left(T_{1}\right), \ldots, \pi\left(T_{n-1}\right)\right\}$ spans $\mathbb{P}^{n}$, and so does $\Omega$.

Let us summarize the results obtained so far. For a general hyperplane section $\Gamma=C \cap \mathbb{P}^{n}$ of $C$ we can find two points $Q_{1}, Q_{2} \in W(\Gamma)$ such that $\Gamma \cup\left\{Q_{1}, Q_{2}\right\}$ is in general linear position. Since $\Gamma$ imposes exactly $2 n+1$ conditions on quadrics [3, p. 36], so does $\Gamma \cup\left\{Q_{1}, Q_{2}\right\}$. Hence $\Gamma \cup\left\{Q_{1}, Q_{2}\right\}$ is a set of $2 n+3$ points in general linear position in $\mathbf{P}^{n}$ which imposes $2 n+1$ conditions on quadrics. Here we use the main ingredient of the proof: a lemma of Castelnuovo states that $\Gamma \cup\left\{Q_{1}, Q_{2}\right\}$ must lie on a rational normal curve $B \subseteq \mathbf{P}^{n}[\mathbf{3}$, p. 36].

Pick a quadric $R$ in $\mathbf{P}^{n}$ which contains $\Gamma$. If $B$ is not contained in $R$ then $2 n+1=$ cardinal of $\Gamma \leq$ cardinal of $(R \cap B)=2 n$, absurd. Hence $B \subseteq R$. Since the ideal of $B$ is generated by quadrics we get $W(\Gamma)=B$. Now recall that $W(\Gamma)=W(C) \cap \mathbf{P}^{n}$. Notice that the above considerations hold for a general hyperplane $\mathbf{P}^{n}$ of $\mathbf{P}^{n+1}$. It follows that $W(C)$ is a surface of minimal degree. $W(C)$ cannot be the Veronese surface in $\mathbf{P}^{5}$ because $C$ has odd degree and is contained in $W(C)$. Therefore $W(C)$ is a rational normal scroll of dimension 2 [3, p. 51]. The homogeneous ideal of $C$ in $\mathbf{P}^{n+1}$ is generated by quadrics and cubics [5] and thus $C$ meets every fiber of $W(C)$ at no more than three points. Next we are going to classify the possible configurations $(W(C), C)$.

Assume first that $W(C)$ is a cone. The vertex $P$ of $W(C)$ must belong to $C$ (otherwise $C$ would have degree $2 n$ or $3 n$ ), and $C$ meets every fiber of $W(C)$ at two other points. Now it is obvious that $C$ is hyperelliptic, and that any hyperplane section of $C$ passing through $P$ belongs to the system $\left|P+n g_{2}^{1}\right|$.

Suppose that $W(C)$ is nonsingular, and denote by $F$ a general fiber of $W(C)$. If $C$ meets $F$ at three points then $C$ is trigonal, and an easy application of the Riemann-Roch formula shows that the divisors of the $g_{3}^{1}$ span lines only when the hyperplane divisor belongs to the system $\left|K_{C}+g_{3}^{1}\right|$. In case $C$ meets $F$ at two points and $H$ denotes a hyperplane divisor of $W(C)$ we have $H^{2}=n, C H=2 n+1$ and $C$ is linearly equivalent to $2 H+b F$. One concludes that $b=1$.

REMARK. By Green-Lazarsfeld's claim, quoted in the Introduction, it follows that in case $W(C)$ is not a scroll and $W(C)$ contains a line, then this line is a trisecant of $C$.

\section{REFERENCES}

1. T. Fujita, Defining equations for certain types of polarized varieties, Complex Analysis and Algebraic Geometry, Cambridge Univ. Press, 1977.

2. M. Green and R. Lazarsfeld, On the projective normality of complete linear series on an algebraic curve, Invent. Math. 83 (1985), 73-90. 
3. J. Harris, $A$ bound on the geometric genus of projective varieties, Ann. Scuola Norm. Sup. Pisa Ser. (4) 8 (1981), 35-68.

4. P. Maroscia and W. Vogel, On the defining equations of points in general position in $\mathbf{P}^{n}$, Math. Ann. 269 (1984), 183-189.

5. B. Saint-Donat, Sur les équations définissant une courbe algébrique, C. R. Acad. Sci. Paris Ser. A 274 (1972), 324-327 and 487-489.

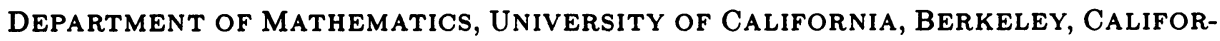
NIA 94720

Permanent address: Departament d'Algebra i Geometria, Facultat de Matemàtiques, Universitat de Barcelona, Gran Via 585, 08007 Barcelona, Spain 